Orthopäde 2015 - 44:275-281

DOI 10.1007/s00132-015-3099-7

Online publiziert: 24. März 2015

(c) Springer-Verlag Berlin Heidelberg 2015
K.-H.Widmer ${ }^{1,2} \cdot$ A. Zich ${ }^{2,3}$

${ }^{1}$ Department für Orthopädie, Universität Basel, Basel, Schweiz

${ }^{2}$ Orthopädische Klinik, Kantonsspital Schaffhausen, Schaffhausen, Schweiz

${ }^{3}$ OSPIDAL, Via da L'Ospidal, Scoul, Schweiz

\title{
Ligamentkontrollierte Positionierung der Knieprothesenkomponenten
}

\begin{abstract}
Eine optimale postoperative Kinematik, verbunden mit einer stabilen, schmerzfreien ligamentären Führung über den ganzen Bewegungsumfang ist das Ziel einer jeden Knieprothetik. Hierfür sind verschiedenste Prothesendesigns und Implantationstechniken entwickelt worden. Diese Studie stellt eine Operationstechnik vor, bei der die Osteotomien intraoperativ zugangsunabhängig ligamentreferenziert vorgenommen werden. Zusammen mit einem kraftkontrollierten Ligament-Tensioner steuern die Ligamente die Osteotomien sequenziell "top-down" und „bottom-up“ gemäß der individuellen Kinematik.
\end{abstract}

\section{Einleitung}

Die Rekonstruktion der individuellen Kinematik eines Gelenkes, wie sie bei der Knietotalprothetik angestrebt wird, erfordert eingehende anatomische und biomechanische Kenntnisse. Die Kinematik eines Gelenkes wird im intakten Zustand einerseits von den Weichteilen, im Wesentlichen den Ligamenten als den passiven und den gelenkübergreifenden Sehnen und Muskeln als den aktiven Zugelementen, und andererseits von den Artikulationsflächen als den mehr oder weniger formschlüssigen Gelenkflächen gesteuert. Bei Gelenkluxationen sind i. d. R die Ligamente und Kapseln rupturiert während die Artikulationsflächen - zumindest makroskopisch - meist intakt sind. Die Gelenkrekonstruktion besteht hier in einer Rekonstruktion der Weichteile, wobei sich der Operateur zur Längenbeurteilung an den Artikulationsflächen orientieren kann. Da- gegen sind bei Gelenkdegenerationen die Artikulationsflächen deformiert. Hier dienen die Ligamente dazu, die Positionierung der Prothesenkomponenten, die die ursprünglichen Artikulationsflächen ersetzen sollen, zu steuern. Bei Luxationsfraktion fehlen beide Referenzmöglichkeiten und müssen vom Operateur intraoperativ erst wieder geschaffen werden.

\section{Operationsstrategien}

In der primären Oberflächenknieendoprothetik haben sich für die Platzierung der Prothesenkomponenten zwei Operationsstrategien etabliert. Einerseits die knochenreferenzierte Positionierung, wie sie von Hungerford propagiert worden ist [14], und andererseits die ligamentreferenzierte Positionierung, die von Insall popularisiert wurde [3, 15]. Für beide Vorgehensweisen werden gleichwertige klinische Resultate berichtet, sodass sich keine eigentliche wissenschaftliche Begründung herausfiltern ließ, die die eine Methode als überlegen gegenüber der anderen herausgestellt hat. In der Literatur werden beiden Strategien sowohl Vor-als auch Nachteile zugeschrieben.

Teil der knöchernen Referenzierungsmethode ist v. a. der Measured-resectionSchritt [21]. Er geht von der Vorstellung aus, dass gerade so viel Knochen reseziert werden muss, wie durch die implantierte Prothese wieder ersetzt wird. Auch wenn darauf abgehoben wird, dass die Messung am nicht deformierten oder „weniger deformierten“ Gelenkabschnitt vorgenommen werden soll, berücksichtigt die Anwendung dieser Methode in Reinform nicht die degenerationsbedingte Defor- mation der Artikulationsflächen und v. a. nicht chronische oder durch die Operation vorgenommene ligamentäre Veränderungen. Es kommt hinzu, dass der etablierte Grundsatz der Orthopädie, dass nämlich die Rekonstruktion bzw. die Korrektur dort vorgenommen wird, wo die Abweichung von der Norm vorliegt, nicht ausreichend berücksichtigt wird, sondern notwendigenfalls die Ligamente an die neuen Artikulationsflächen angepasst werden, damit das Balancing erreicht wird [20, 22].

Dagegen ist die ligamentreferenzierte Operationstechnik vor allem durch das "gap balancing“ charakterisiert. Darunter versteht man die Äquilibrierung der knöchernen Lücken in Streckung und in $90^{\circ}$-Flexion, die entstehen, wenn die Ligamente mit einem Tensioner angespannt werden, und am Femur und an der Tibia zusammengenommen so viel Knochen reseziert wird, dass der aufgespannte Spalt jene Höhe besitzt, der zur Aufnahme beider Komponenten freigestellt werden muss. Die Spaltweiten sollen in Extension und Flexion gleich sein, wobei in Flexion lateralseitig eine um 1-2 mm weitere Öffnung akzeptiert wird.

Diese zwei Strategien dominieren im Wesentlichen bis heute die Implantationstechniken in der Knieendoprothetik mit unwesentlichen Modifikationen, was nicht verwundert, da sie jeweils auf ihre Weise versuchen, die beiden kinematikbestimmenden Anteile, d. h. die Artikulationsflächen und die ligamentäre Führung aufeinander abzugleichen $[6,7,23]$. Stark verkürzt könnte man sagen, dass bei der knochenreferenzierten Knieprothesenimplantation der resezierte Knochen ein- 


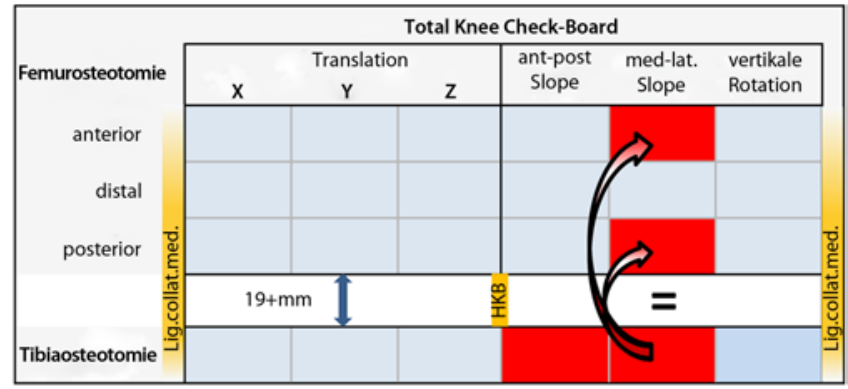

Abb. $1<$ Das, "Total Knee Check-Board" (HKB hinteres Kreuzband)

fach ersetzt und die Ligamente den „neuen“Artikulationsflächen anpasst werden, während bei der ligamentreferenzierten Implantationstechnik die ligamentäre Führung der Ligamente erhalten bleibt und die Artikulationsflächen dort platziert werden, wohin sie durch die neue ligamentäre Führung geleitet werden.

\section{Kinematik des Knies}

Die ligamentäre Führung des Kniegelenkes ist postoperativ bei allen heute gebräuchlichen Operationstechniken verändert. Selbst bei der aktuell von Stephen Howell [13] propagierten und als „kinematically aligned“ bezeichneten Implantationstechnik wird das vordere Kreuzband reseziert, und so ist die von Phil Noble (ICJR 2014) gemachte Bemerkung, dass nämlich ,the best what you can get is an ACL-deficient knee", mehr als berechtigt. Damit einhergehend muss auch die Frage gestellt werden, ob die „physiologische" Kinematik, die allenthalben als das ultimative Ziel einer perfekten Knieendoprothetik genannt wird, überhaupt ein sinnvolles und/oder realistisches Ziel darstellen kann. Die vielen detaillierten anatomischen, biomechanischen, ganganalytischen, geometrischen, simulierenden, stabilitätstestenden und oft sehr fokussierten Bewegungsuntersuchungen [16] haben neben den zweifellos handfesten nachhaltigen wissenschaftlichen Ergebnissen auch gezeigt, dass das menschliche Kniegelenk zwar einigen biomechanischen Grundsätzen folgt, die auch Eingang in die prothetischen Operationstechniken und Prothesendesigns gefunden haben, dass aber im individuellen Fall kleine Normabweichungen vorkommen, die bei der unreflektierten Anwendung eines Standardprozederes dazu beitragen können, dass nur ein befriedigen- des oder gar unbefriedigendes und nicht das gewünschte gute oder sehr gute Operationsergebnis zustande kommt.

\section{) Die Kinematik fesselte mehrere Generationen von Orthopäden}

Als Operateur hat man es sprichwörtlich in der Hand, die individuellen mechanischen Konditionen eines Kniegelenkes während des Operationsablaufs fortlaufend zu ergründen und zu gestalten. Die Beschäftigung mit der Kinematik des Kniegelenks hat deshalb bereits mehrere Generationen von Orthopäden gefesselt, was gleichermaßen für das natürliche wie für das prothetisch versorgte Kniegelenk gilt $[1,2,9,13]$.

Die Kinematik beschreibt die (dreidimensionale) Geometrie der Bewegung eines Körpers relativ zu einem anderen. Beim Kniegelenk bewegen sich zwei (Fest-)Körper zueinander: Femur und Tibia [8]. Zur Analyse deren Kinematik empfiehlt es sich sehr, die traditionelle Sichtweise der Anatomen beizubehalten und das Femur als "punctum fixum“ und die Tibia als „punctum mobile“ zu betrachten. Dies entspricht auch viel eher der intraoperativen Perspektive des Operateurs und macht die Terminologie klarer, eindeutiger und besser nachvollziehbar.

In dieser Sichtweise „umkreist“ der mobile Unterschenkel das festgehaltene Femur, d. h. Tibia und Fibula sind sozusagen auf dem „Orbit“ um die Femurkondylen. Die Ligamente lenken dabei den Unterschenkel, indem sie die „Flugbahn“ und die „Flughöhe“ bestimmen und bei der Flexions- oder Extensionsbewegung in begrenztem Masse Rotationen im Sinne von Innen- bzw. Außenrotationen oder
Varus- bzw. Valgusabweichungen zulassen bzw. auslösen. Idealerweise wird die ligamentäre Führung durch zwei Artikulationsflächen ergänzt, deren Oberflächengeometrien sich in allen Gelenkstellungen komplementartig in diese Führung einpassen. Artikulationsflächen und Ligamentführung sind damit zwei Gelenkcharakteristika, die zusammenspielen müssen, damit ein Gelenk gut funktionieren kann [5, 17].

Die Form des natürlichen Kniegelenks bildet sich im Verlauf des Wachstums im Normalfall so aus, dass sich eine aufeinander abgestimmte Symbiose aus passenden Artikulationsflächen und Ligamentführungen entwickelt. Bei der Arthroseentwicklung hat sich diese im Laufe der Jahre entzweit und der Operateur steht bei der Knietotalprothesenimplantation vor der nicht ganz leichten Aufgabe, eine vom Prothesenhersteller gekaufte Oberflächengeometrie in eine ihm im Detail nicht genau bekannte Ligamentführung einzusetzen [19], um diese Symbiose wiederherzustellen. Es ist offensichtlich, dass je nach operativen Randbedingungen dabei nur eine mehr oder weniger optimale Annäherung an den Idealzustand möglich ist.

\section{Das „Total Knee Check-Board“}

Die gegenwärtigen Knieprothesenmodelle erfordern prinzipiell alle die gleichen Osteotomien an Femur und Tibia, die sich natürlich wegen der verschiedenen Prothesendesigns leicht unterscheiden. Am Femur werden 3 Hauptosteotomien und an der Tibia deren eine vorgenommen. Diese 4 Osteotomien sind voneinander abhängig. Die Abhängigkeiten können in einer Tabelle aufgeführt werden, die hier als „Total Knee Check-Board“ bezeichnet wird (• Abb. 1). In den Zeilen sind die 4 Osteotomien mit ihren je 6 Freiheitsgraden aufgeführt. Die gegenseitigen Abhängigkeiten bewirken, dass die Festlegung eines Freiheitsgrades bei einer Osteotomie die Freiheitsgrade einer davon abhängigen blockiert. Die direkte Abhängigkeit der 3 Femurosteotomien untereinander ist durch die Femurkomponente gegeben. Die Freiheitsgrade dieser Osteotomien werden aber infolge des ligamentären Links auch durch die Tibiaos- 
teotomie beeinflusst. Beispielsweise wird bei der Tibia-first-Operationstechnik mit der Tibiaosteotomie („first“) auch die Lage der Femurosteotomien definiert und damit in einem sehr frühen Stadium der Prothesenimplantation die Komponentenpositionierung und -größe festgelegt. Die Analyse der Operationsschritte mithilfe des „Total Knee Check-Boards“ führt u. a. auch dazu, dass die Größe der Femurkomponente auch als variabel angesehen werden kann und nicht bereits präoperativ fest geplant werden muss, sondern sich erst intraoperativ zu einem späten Operationszeitpunkt definitiv ergibt.

\section{Ligamentkontrollierte Prothesenpositionierung}

Obschon bei der ligamentkontrollierten Prothesenpositionierung die steuernde Funktion der Ligamente im Zentrum steht, beginnen auch diese Operationen mit einer Osteotomie, typischerweise ist dies die Tibiaosteotomie, wie auch bei der hier vorgestellten Methode. Allerdings wird diese hier lediglich präliminär vorgenommen, wobei die Orientierung, d. h., der posteriore und der mediolaterale "Slope“, aus der präoperativen Planung entnommen werden und endgültig sind. Lediglich das Level dieser Tibiaosteotomie liegt proximaler und ist gerade so gewählt, dass der notwendige Platz für den Tensioner geschaffen wird. Nach bzw. mit den Ligament-release-Schritten wird in Streckung die Beinachse orthograd justiert und in $90^{\circ}$-Flexion durch Aufspannen die Rotation der Femurkomponente ligamentkontrolliert eingestellt. Als erste definitive Femurosteotomie wird so die anteriore vorgenommen, wobei deren Flexion/Extension-Stellung mit dem intramedulären Stab und die Rotation mit dem Tensioner parallel zur Tibiaosteotomie und tangential zur anterioren Femurkontur ausgerichtet wird (• Abb. 2a, b). Die visuelle Kontrolle vermeidet so sehr einfach ein „notching“. Als nächste Osteotomie folgt die distale Femurosteotomie, deren Varus-/Valgus-Orientierung und kraniokaudales Level entsprechend der präoperativen Planung einge-

Orthopäde 2015 - 44:275-281 DOI 10.1007/s00132-015-3099-7

(c) Springer-Verlag Berlin Heidelberg 2015

\section{K.-H. Widmer · A. Zich}

\section{Ligamentkontrollierte Positionierung der Knieprothesenkomponenten}

\section{Zusammenfassung}

Hintergrund. Ziel jeder Knietotalprothesenimplantation ist die optimale postoperative Kinematik, verbunden mit einer stabilen ligamentären Führung über den ganzen Bewegungsumfang des operierten Kniegelenkes, damit sich der Patient wieder schmerzfrei bewegen kann. Um dieses postoperative Ziel zu erreichen, sind im Rahmen der Entwicklung der Knietotalprothetik verschiedenste Prothesendesigns und Implantationstechniken entwickelt worden.

Fragestellung. Diese Studie stellt eine Operationstechnik vor, bei der die Osteotomien anhand definierter Referenzen präoperativ geplant und intraoperativ sowohl knochenreferenziert als auch ligamentgesteuert festgelegt werden.

Methode. Diese Implantation kann prinzipiell über jeden Zugang erfolgen und bei kreuzbanderhaltenden wie bei -ersetzenden Techniken eingesetzt werden. Nach Eröffnen des Gelenkes und ersten Releaseschritten werden die Osteotomien sequenziell vorgenommen, beginnend mit der anterioren und distalen Femurosteotomie, die gemäß der präoperativen Planung und mit ligamentkontrollierter Rotation ausgeführt werden. Es folgt die Justierung des Levels der Tibiaosteotomie im Sinne einer Top-down-Referenzierung. Der Beugespalt wird bottom-up-referenziert. Die beiden Osteotomiespalthöhen werden jeweils mit einem kraftkontrollierten Tensioner gemessen.

Diskussion. Diese Operationstechnik stellt eine gemischte ossär- und ligamentbasierte Vorgehensweise dar, die die veränderten $\mathrm{Ar}$ tikulations- und Ligamentverhältnisse intraoperativ schrittweise berücksichtigt und so auf die neue, durch die Prothetik veränderte Kniekinematik eingeht. Wünschenswert wäre allenfalls noch die intraoperative Erfassung der individuellen Kinematik mittels adaptierter Navigation.

\section{Schlüsselwörter}

Bewegungsumfang $\cdot$ Kinematik .

Knietotalprothetik $\cdot$ Kniearthroplastik .

Ligamente

\section{Ligament-controlled positioning of the knee prosthesis components}

\section{Abstract}

Background. There are at least two predominant goals in total knee replacement: first, the surgeon aims to achieve an optimal postoperative kinematic motion close to the patient's physiological range, and second, he aims for concurrent high ligament stability to establish pain-free movement for the entire range of motion. A number of prosthetic designs and surgical techniques have been developed in recent years to achieve both of these targets.

Objectives. This study presents another modified surgical procedure for total knee implantation. As in common practice the osteotomies are planned preoperatively, referencing well-defined bony landmarks, but their placement and orientation are also controlled intraoperatively in a stepwise sequence via ligamentous linkages.

Method. This method is open to all surgical approaches and can be applied for PCL-conserving or -sacrificing techniques. The anteri- or femoral osteotomy is carried out first, followed by the distal femoral osteotomy. Then, the extension gap is finalized by tensioning the ligaments and "top-down" referencing at the level of the tibial osteotomy, followed by finishing the flexion gap in the same way, except that the osteotomy of the posterior condyles is referenced in a "bottom-up" fashion. Discussion. Hence, this technique relies on both bony and ligament-controlled procedures. Thus, it respects the modified ligamentous framework and drives the prosthetic components into the new ligamentous envelope. Further improvement may be achieved by additional control of the kinematics during surgery by applying modern computer navigation technology.

\section{Keywords}

Kinematics · Knee Arthroplasty - Ligaments . Range of Motion · Total Knee Replacement 

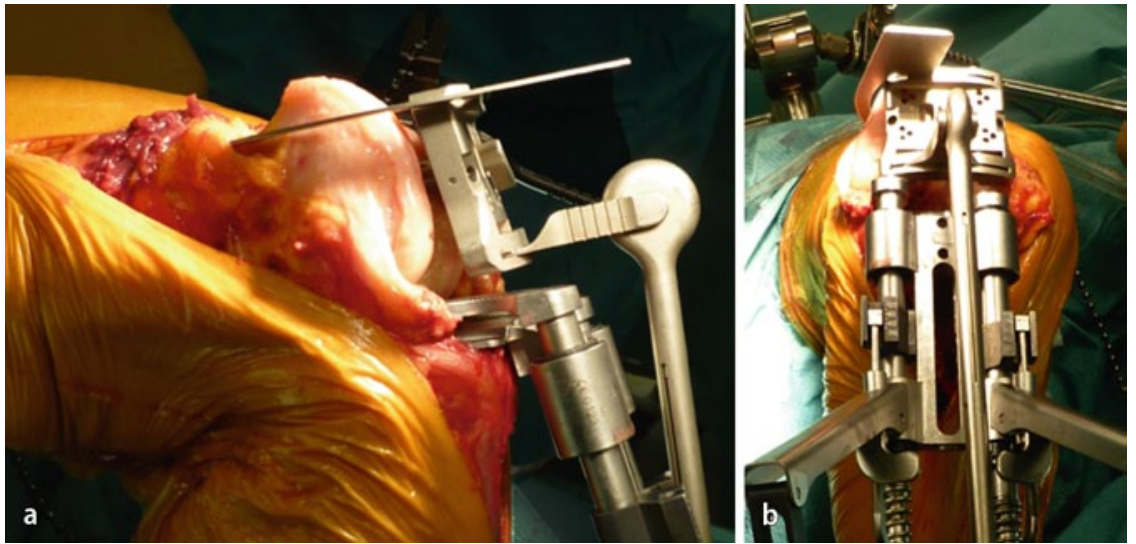

Abb. $2 \Delta$ Einstellung des Schnittblockes zur tangentialen anterioren Femurosteotomie mit ligamentkontrollierter Rotation, a laterale und $\mathbf{b}$ anteriore Ansicht
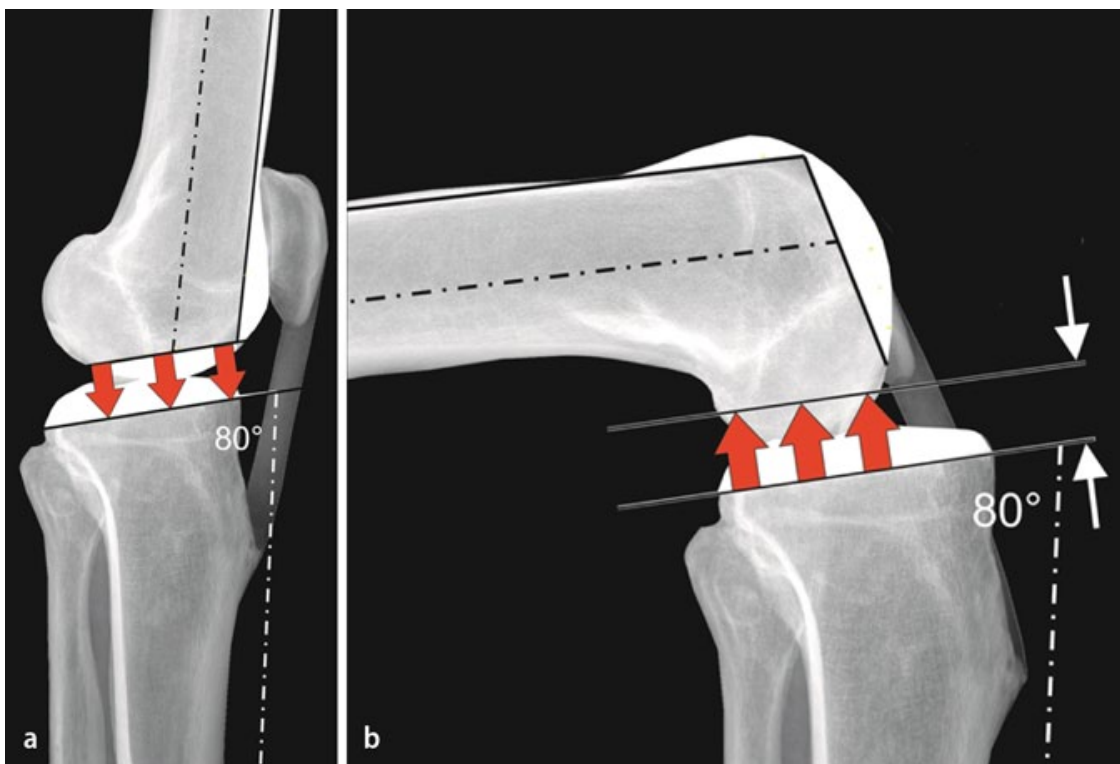

Abb. $3 \Delta$ a Top-down-Referenzierung der Tibiaosteotomie in Streckstellung und b Bottom-up-Referenzierung der posterioren Femurosteotomie in $90^{\circ}$-Flexion

stellt werden. Mit dieser zweiten Osteotomie sind die Komponentenpositionen sowohl am Femur- als auch an der Tibia im Grunde festgelegt.

Jetzt wird das Knie in die Streckstellung gebracht, der Streckspalt mit dem Tensioner aufgespannt und die Tibiaosteotomie per Top-down-Referenzierung, ausgehend von der distalen Femurosteotomie, auf das definitive Niveau vorgenommen ( $\mathbf{A} \mathbf{A b b}$. 3a). Daraufhin wird das Knie in die $90^{\circ}$-Flexion gebracht, der Beugespalt mit dem Tensioner aufgespannt und die posteriore Femurosteotomie per Bottom-up-Referenzierung festgelegt (• Abb. 3b). Diese Osteotomie wird für die nächstpassende, d. h., die nächstklei-

\section{Limitationen der derzeitigen Methode}

Alle kinematikbasierten Implantationsmethoden bräuchten eigentlich intraoperative kinematikfähige dreidimensionale Messverfahren, damit sie sich wirklich komplett praktisch umsetzen lassen. Die oben beschriebene manuelle Vorgehensweise stellt einen Kompromiss dar in Ermangelung solch eines geeigneten dreidimensionalen kinematischen Messverfahrens. Potenziell würden gebräuchliche Computernavigationssysteme die gewünschten 3-D-Informationen liefern können. Anwendungsfertige Verfahren stehen unseres Wissens aktuell für den intraoperativen Einsatz allerdings nicht zur Verfügung.

\section{Vorläufige Erfahrungen und klinische Zwischenergebnisse}

Seit 2010 ist bei mehr als 130 Patienten die Knietotalprothesenimplantation mit dieser Operationsmethode vorgenommen worden. Im Rahmen einer Zwischenauswertung wurden die Daten der Patienten mit einem mindestens 1-jährigen postoperativen Verlauf klinisch und radiologisch ausgewertet.

Die minimal angestrebte Flexion von $120^{\circ}$ wurde von $86 \%$ der Patienten erreicht oder übertroffen, bei den übrigen Patienten ist dieses Ziel zum Untersuchungszeitpunkt noch nicht erreicht worden (• Abb. 4). Bei der klinischen Untersuchung wurden alle Kniegelenke als ligamentär stabil über den ganzen Bewegungsumfang beurteilt.

Die beiden radiologischen Verlaufskontrollen 6 Wochen und $1 \mathrm{Jahr}$ postoperativ zeigten, dass die geplante Positionierung der beiden Prothesenkomponenten erreicht worden ist. Speziell hat sich ergeben, dass in vielen Fällen ein kleiner, meist nur $1^{\circ}$ großer medialer "slope“ der Tibiakomponente gegenüber der mechanischen Tibiaachse resultierte ( $\bullet$ Abb. 5). Diese Stellung wird durch die femorale Komponente mit einer Valgisation und (verminderten) Außenrotation von gleicher Größe beantwortet.

Die Beinachse wurde nur orientierend klinisch beurteilt und postoperativ nicht mit Ganzbeinaufnahmen kontrolliert, 

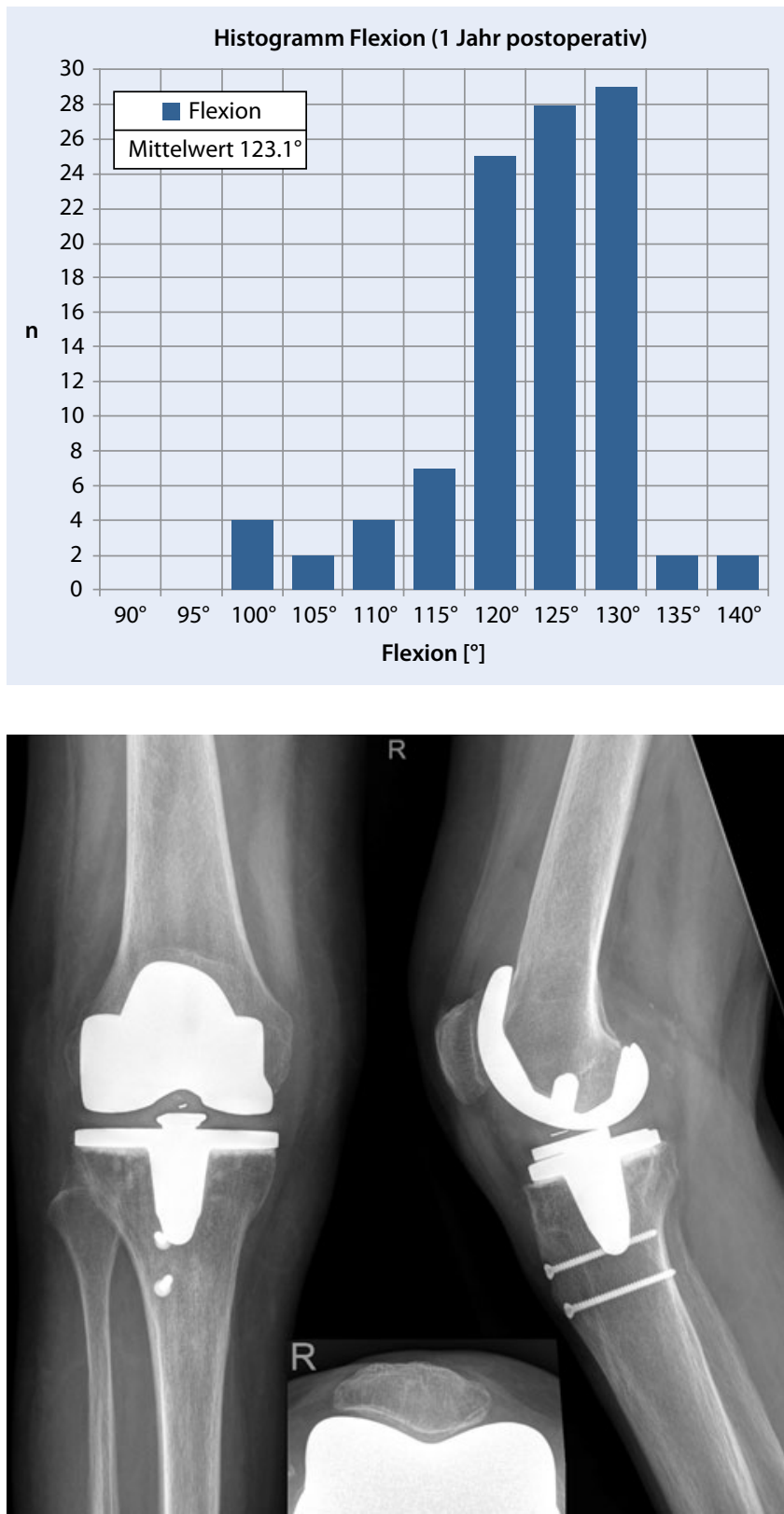

Abb. $4<$ Flexionsfähigkeit ein Jahr postoperativ

Abb. $5<$ Röntgenkontrolle 1 Jahr postoperativ mit LCS-APG-Knieprothese und konsolidierter Tuberositasosteotomie

weshalb quantitative Aussagen zur Beinachse nicht gemacht werden. Die Ganzbeinaufnahme ist jedoch Teil der präoperativen Planung.

\section{Rekonstruktion des posterioren Femurkondylenindexes}

Die Rekonstruktion des posterioren Femurkondylus stellt generell und speziell bei der vorgestellten Operationsmethode ein entscheidendes Performancemerkmal dar $[4,18,21]$. Damit ein Vergleich der Größe dieses Offsets innerhalb und zwischen den Patienten möglich ist, wurde eine Indexierung des absolut gemesse-

\section{Patella-Tracking}

Das Patella-Tracking wird häufig diskutiert und ebenso häufig wird das „maltracking“ als Ursache für den anterioren postoperativen Knieschmerz angegeben. Die geschilderte Operationsmethode vermeidet eine zu exzessive Außenrotation der Femurkomponente und bietet wohl zusammen mit dem Design der benutzten LCS-Prothese eine solide prothetische Führung der Patella, da bisher nur von einer Patientin über einen vorderen Knieschmerz geklagt wurde, sodass auch in diesem Kriterium ein akzeptables Resultat erreicht worden ist. Möglicherweise hat hierzu der von We. Müller eingeführte laterale Subvastuszugang mit Tuberositasosteotomie beigetragen [12], der bei fast allen Implantationen angewandt worden ist.

\section{Diskussion}

Die ligamentkontrollierte Knietotalprothesenimplantation richtet die Positionierung der Prothesenkomponenten an den Ligamenten aus. Voraussetzung dass dieses Vorgehen zum richtigen Ziel führt, ist, dass $\mathrm{zu}$ einem frühen Operationszeitpunkt, d. h., vor der ersten endgültigen Osteotomie, die Ligamente „released“ werden. Die Ligamente müssen ihre Führungsfunktion ausüben können, ohne dass diese Funktion durch Osteophyten, hypertrophe Weichteile oder kontrakte Kapselanteile eingeschränkt wird. Die Ligamente müssen außerdem „kompetent" sein, d. h., sie müssen ihrer Stabilisierungs- und Führungsaufgabe noch gewachsen sein, beispielsweise kann eine vorangegangene ligamentäre Verletzung die Anwendung der ligamentkontrollierten Operationstechnik u. U. ausschließen.

Im Gegensatz dazu setzt die knochenreferenzierte Implantationstechnik nicht unbedingt die gleiche Ligamentfunktion voraus und gleicht notwendigenfalls die Ligamente gegen Ende der Operation den prothetischen Artikulationsflächen an. Das knochenreferenzierte Vorgehen ist unter den Operateuren deutlich beliebter. Außerdem lässt es sich viel einfacher in ein Operationsinstrumentarium umsetzen, da die knöchernen Referenzen im wahrsten Sinne des Wor- 


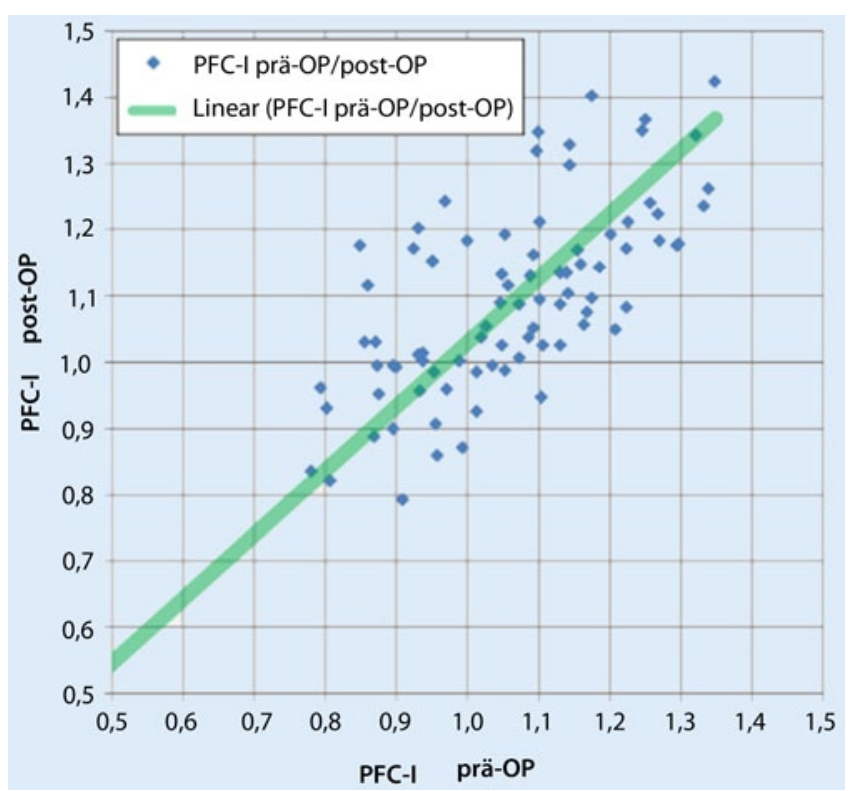

Abb. $6<$ Individuelle Rekonstruktion des posterioren femoralen Kondylenindex (PFC-I) tes einfacher fassbar sind. Bei der besagten Anpassung („release“) werden die Ligamente entweder elongiert oder im Weichteilverbund abgelöst. Ein Nachteil kann sich unter Umständen dadurch ergeben, wenn daraus eine ligamentäre Instabilität resultiert. In jedem Fall berücksichtigt dieses Vorgehen nicht die individuelle Gelenkkinematik, sondern gleicht die Ligamente dem durch das Prothesensystem vorgegebenen Artikulationsstandard an $[10,11]$.

Das ligamentkontrollierte Vorgehen beseitigt ursachengerecht die durch die Arthrose verursachte Deformität der Gelenkflächen und fügt die neuen prothetischen Artikulationsflächen passend in den veränderten "ligamentous frame“ ein. Folgerichtig ist bei der konsequenten Anwendung dieser Methode ein Eingriff in die ligamentäre Führung nur in Ausnahmefällen notwendig. Selbst bei großen, mehr als $15^{\circ}$ messenden Achsdeformitäten können die Prothesenkomponenten ligamentkontrolliert positioniert werden, wenn die Ligamente richtig „released“ werden und sie die Tibia im gesamten Bewegungsumfang stabil um das Femur führen können. Anders ausgedrückt ist die Häufigkeit von Ligamentanpassungen mit dieser Operationstechnik auf ein Minimum zurückgegangen und wird eigentlich nur noch vorgenommen, wenn es sonst nicht gelingt, die mechanische Beinachse in den $\pm 3^{\circ}$-Korridor um die Mikulizc-Linie einzujustieren.
Die ligamentkontrollierte Implantationstechnik setzt keine Bewegungsachsen voraus. Diese Technik akzeptiert die Bewegungsbahn der Tibia, wie sie sich durch die ligamentäre Führung ergibt. Insofern unterscheidet sie sich von der Howell'schen kinematischen Prothesenpositionierung, die Anleihen an der Measured-resection-Technik macht und die Bewegungsachsen definiert. Mit der gegenwärtigen hier dargelegten manuellen Vorgehensweise wird die korrekte Abstimmung der Artikulationsflächen auf die dreidimensionale Bewegungsbahn nur an zwei Stellen kontrolliert: in $0^{\circ}$-Extension und $90^{\circ}$-Flexion. Es wäre wünschenswert und theoretisch auch zu fordern, dies an mehreren Flexionsstellungen oder gar kontinuierlich über den ganzen Bewegungsspielraum zu tun. In jedem Fall lassen sich intraoperativ die ermittelten Komponentenpositionen im Sinne einer vergleichenden Kontrolle natürlich mit den bekannten achs- und knochenreferenzierten Referenzen vergleichen, wie beispielsweise der transepikondylären Achse, der Whiteside-Linie, den Meniskusbasen oder der proximalen PCL-Insertion.

Da die Bewegungsachsen selbst nicht gebraucht werden, entfällt deren diskussionsreiche intraoperative Suche. Die Geometrie der Kinematik von multipel geführten Körpern kennt ohnehin nur eine momentane Rotationsachse, sodass sich viele, allerdings nur „momentan“ gültige,
Antworten für die Bewegungsachse finden ließen.

Mit der ligamentkontrollierten Implantationstechnik gelingt es zielgerichtet, Standardimplantate, die „Standardartikulationsflächen “ tragen, möglichst optimal in den individuellen "ligamentous frame“ einzusetzen. Ein neuerer Ansatz geht einen vergleichbaren Weg und setzt allerdings an der anderen Stelle an: die Technik der Individualprothesen (Fa. ConforMIS, Fa. Symbios) rekonstruiert die individuelle präarthrotische, nicht deformierte anatomische Artikulationsfläche und setzt diese knochenreferenziert mit patientenspezifischen Schnittblöcken an die präoperativ bestimmte anatomische Position ein, in der Annahme, dass die Ligamente durch die rekonstruierten Artikulationsflächen ebenfalls wieder aufgerichtet werden und die frühere Führungsfunktion wieder aufnehmen. Dieses Vorgehen ist sehr attraktiv, wobei lediglich kritisch angemerkt werden sollte, dass bei den aktuellen Operationstechniken der „ligamentäre Rahmen “ durch das Fehlen des vorderen Kreuzbandes nicht mehr derselbe wie vor der Prothesenimplantation ist. Zum Ausgleich kann die intraoperative individuelle Anpassung der Polyethyleninlayhöhen an die mediale und laterale Gelenkspaltweiten dieser Veränderung Rechnung tragen. So kann die neue ligamentäre wieder der rekonstruierten früheren artikulären Kinematik angeglichen werden.

\section{Fazit für die Praxis}

- Es besteht kein Zweifel, dass die individualisierten Operationstechniken im Kontext der individualisierten Medizin zu sehen sind und sie ein profundes Potenzial bieten, die operativen Behandlungen auch in der Prothetik zu verbessern.

- Sie erfordern allerdings die Bereitschaft, die Individualitäten des jeweiligen Patienten prä- und/oder intraoperativ zu ergründen, was mit einem zeitlichen Mehraufwand verbunden ist.

- Die berechtigte Erwartung, dass speziell unsere Kniepatienten davon in mehrfacher Weise profitieren werden, sollte genügend Motivation für uns Operateure sein. 


\section{Korrespondenzadresse}

PD Dr.med. Dipl.Ing. K.-H.
Widmer
Department für Orthopädie
Universität Basel
Spitalstrasse 21,4031 Basel
Karl-Heinz.Widmer@unibas.ch;
Karl-Heinz.Widmer@
spitaeler-sh.ch

\section{Einhaltung ethischer Richtlinien}

Interessenkonflikt. K.-H. Widmer und A. Zich geben an, dass keine Interessenkonflikte bestehen.

Dieser Beitrag beinhaltet keine Studien an Menschen oder Tieren.

\section{Literatur}

1. Andriacchi TP, Galante JO, Fermier RW (1982) The influence of total knee-replacement design on walking and stair-climbing. J Bone Joint Surg Am 64(9):1328-1335

2. Aalbersberg S et al (2005) Orientation of tendons in vivo with active and passive knee muscles. J Biomech 38(9):1780-1788

3. Aunan E et al (2012) A new method to measure ligament balancing in total knee arthroplasty: laxity measurements in 100 knees. Arch Orthop Trauma Surg 132(8):1173-1181. doi: 10.1007/s00402-0121536-1

4. Bellemans J, Robijns F, Duerkinckx J et al (2005) The influence of tibial slope on maximal flexion after total knee arthroplasty. Knee Surg Sports Traumatol Arthrosc 13:193-196

5. Bull AM et al (2008) Changes in knee kinematics reflect the articular geometry after arthroplasty. Clin Orthop Relat Res 466(10):2491-2499. doi:10.1007/s11999-008-0440-z

6. Dennis DA, Komistek RD, Kim RH et al (2010) Gap balancing versus measured resection technique for total knee arthroplasty. Clin Orthop Relat Res 468(1):102-107

7. Eckhoff DG et al (2005) Three-dimensional mechanics, kinematics, and morphology of the knee viewed in virtual reality. J Bone Joint Surg Am 87(Suppl 2):71-80 (No abstract available)

8. Eckhoff DG et al (2005) Three-dimensional-mechanics, kinematics and morphology of the knee viewed in virtual reality. J Bone Joint Surg Am 87(Suppl 2):71-80

9. Hagemeister $\mathrm{N}$ et al (2005) A reproducible method for studying three-dimensional knee kinematics. J Biomech 38(9):1926-1931

10. Hanada $\mathrm{H}$ et al (2007) Bone landmarks are more reliable than tensioned gaps in TKA component alignment. Clin Orthop Relat Res 462:137-142

11. Hanada $H$, Whiteside LA, Steiger J, Dyer P, Naito $M$ (2007) Bone landmarks are more reliable than tensioned gaps in TKA component alignment. Clin Orthop Relat Res 462:137-142

12. Hirschmann MT et al (2010) Anterolateral approach with tibial tubercle osteotomy versus standard medial approach for primary total knee arthroplasty: does it matter? BMC Musculoskelet Disord 22(11):167. doi:10.1186/1471-2474-11-167
13. Howell SM, Papadopoulos S, Kuznik KT, Hull ML (2013).Accurate alignment and high function after kinematically aligned TKA performed with generic instruments. Knee Surg Sports Traumatol Arthrosc 21(10):2271-2280. doi:10.1007/s00167013-2621-x

14. Hungerford DS, Kenna RV, Krackow KA (1982) The porous-coated anatomic total knee. Orthop Clin North Am 13(1):103-122

15. Insall JN et al (1993) The gap technique. In: Insall JN (Hrsg) Surgery of the knee, 2. Aufl. Churchill Livingstone, New York

16. Marra MA et al (2015) A subject-specific musculoskeletal modeling framework to predict in vivo mechanics of total knee arthroplasty. J Biomech Eng 137(2). doi:10.1115/1.4029258

17. McCalden RW et al (2010) The role of polyethylene design on postoperative TKA flexion: an analysis of 1534 cases. Clin Orthop Relat Res 468(1):108-114. doi:10.1007/s11999-009-1127-9

18. Mitsuyasu $\mathrm{H}$ et al (2011) Enlarged post-operative posterior condyle tightens extension gap in total knee arthroplasty. J Bone Joint Surg Br 93(9):1210 1216. doi:10.1302/0301-620X.93B9.25822

19. Nakahara H et al (2012) Sagittal cutting error changes femoral anteroposterior sizing in total knee arthroplasty. Clin Orthop Relat Res 470(12):3560 3565. doi:10.1007/s11999-012-2397-1

20. Sabbioni G et al (2011) Gap balancing versus measured resection technique using a mobile-bearing prosthesis in computer-assisted surgery. Musculoskelet Surg 95(1):25-30. doi:10.1007/s12306-0110110-2

21. Tsukeoka T, Lee TH (2012) Sagittal flexion of the femoral component affects flexion gap and sizing in total knee arthroplasty. J Arthroplasty 27(6):10941099. doi:10.1016/j.arth.2011.10.015

22. Whiteside LA (1995) Ligament release and bone grafting in total arthroplasty of the varus knee. Orthopedics 18(2):117-123. doi:10.3928/0147-744719950201-09

23. Wyss T et al (2008) Tension controlled ligament balanced total knee arthroplasty: 5 -year results of a soft tissue orientated surgical technique. Arch Orthop Trauma Surg 128(2):129-135

\section{Hier steht eine Anzeige.}

